\title{
ELECTRORREMEDIACIÓN DE CADMIO Y NÍQUEL DE PASIVOS AMBIENTALES DE SUELO CONTAMINADO POR DERRAMES DE PETRÓLEO
}

\section{Electrorremediation of cadmium and nickel of environmental liabilities in contaminated soils by petroleum spills}

\author{
${ }^{1}$ Fausto Yaulema Garcés, ${ }^{1}$ Andrés Beltrán, ${ }^{1}$ Jenner Baquero, ${ }^{1}$ Cristian Carrera, ${ }^{1,2}$ Alex Gavilanes \\ Montoya*
}

${ }^{1}$ Escuela Superior Politécnica de Chimborazo, Riobamba, Ecuador

${ }^{2}$ Universidad de Transilvania de Brasov, Brasov, Rumania

*a_gavilanes@espoch.edu.ec

En el presente artículo se presenta las acciones y resultados de la electroremediación por derrames de petróleo en el cantón Joya de los Sachas, Ecuador. Las acciones para la electrorremediación de metales pesados como el cadmio y níquel de los pasivos ambientales de suelo contaminado, generado por derrames de petróleo en el cantón Joya de los Sachas (sector la Parker) consistió en la determinación de las características físicoquímicas y mineralógicas del suelo, además, la identificación de los potenciales óptimos para su remediación durante $48 \mathrm{~h}$ a través de soluciones electrolíticas para inmovilizar iones metálicos y el análisis de los fenómenos de transporte de los metales pesados en base a los estudios de espectroscopia de absorción atómica y microscopia de barrido electrónico. Estas acciones, fueron determinantes para definir que los procesos electrocinéticos son más efectivos en suelos arcillosos debido a su carga superficial negativa. Como resultados más importantes se tiene que para el cadmio de 25,55 ppm, permitiendo alcanzar una eficiencia máxima de remoción de 93,71\%, para el níquel una concentración de 37,20 ppm alcanzando una eficiencia del 85,12\%, permitiendo cumplir con la normativa aplicable para actividades hidrocarburíferas. Finalmente, mediante la técnica de microscopia de barrido electrónico se pudo constatar una modificación de los elementos iniciales, que también se vieron desplazados por efectos del campo eléctrico.

Palabras claves: Electrorremediación, Joya de los Sachas, Hidrocarburos, Pasivos ambientales, Cadmio, Níquel.

The electroremediation of heavy metals such as cadmium and nickel, from the environmental liabilities of contaminated soil, generated by oil spills in the canton Joya de los Sachas, la Parker sector, consisted of determining the physical-chemical and mineralogical characteristics of the soil; the identification of the optimal potentials for its remediation during 48 hours through electrolytic solutions to immobilize metal ions and the analysis of heavy metal transport phenomena based on the studies of atomic absorption spectroscopy and electron scanning microscopy; which were determinant to define that the electrokinetic processes are more effective in clay soils due to their negative surface charge. For the cadmium of $25.55 \mathrm{ppm}$, allowing a maximum removal efficiency of $93.71 \%$, for nickel a concentration of $37.20 \mathrm{ppm}$ reaching an efficiency of $85.12 \%$, allowing compliance with the applicable regulations for hydrocarbon activities. Finally, by means of the electronic scanning microscopy technique, we can observe a modification of the initial elements, which were also displaced by effects of the electric field.

Key words: Electroremediation, Joya de los Sachas, Hydrocarbons, Environmental liabilities, Cadmium, Nickel 


\section{INTRODUCCIÓN}

En el Ecuador la contaminación por metales pesados se debe principalmente a las actividades mineras (1) e hidrocarburíferas (2), en las principales provincias donde hay diferentes procesos de exploración (3), explotación, transporte (4) y refinación de hidrocarburos (5), accidentalmente se producen roturas en las tuberías causando derrames de petróleo que contaminan el agua, suelo, flora y fauna (6), causando graves problemas ambientales y socioeconómicos (7).

En el sector La Parker cantón Joya de los Sachas, las distintas actividades petroleras cada año generan miles de toneladas de contaminantes sólidos, líquidos y gaseosos (8) en concentraciones mayores a las permitidas por las normativas ambientales vigentes (9), y sin que sobre estos se haya aplicado un correcto tratamiento de remediación (10), generando significativos pasivos ambientales (11).

En el contexto nacional, existen escasos estudios en relación a la remediación de suelos contaminados por metales pesados como el cadmio (Cd) y níquel (Ni) (12).

La exposición a las condiciones climáticas prevalentes del lugar, puede ocasionar una propagación de los contaminantes por medios físicos (13), o bien una transformación de los residuos con el consecuente desplazamiento químico de los elementos potencialmente tóxicos (14), como la lixiviación de metales pesados que alteran la estructura del suelo y los niveles freáticos del agua (15), así afectando al entorno ambiental y la salud de la población expuesta (16)

En los tratamientos físicos y químicos la electrorremediación es una tecnología emergente que se viene desarrollado desde las últimas 3 décadas (17) (18) y prometedora como es el caso como es el caso de la remoción de plomo $(\mathrm{Pb})$, cercana a un $100 \%$ de eficiencia (19) que aprovecha las propiedades conductivas del suelo, cuyo objetivo es separar y extraer los contaminantes orgánicos e inorgánicos presentes en suelos, lodos y sedimentos contaminados (20). Aunque también se ha aplicado en la remoción de cromo, cadmio, mercurio, plomo, manganeso, zinc y arsénico (21-24)

En este estudio, se determinaron las características físicoquímicas y mineralógicas del suelo contaminado (25), se identificaron los potenciales óptimos para la remediación de metales pesados (26) y finalmente se analizaron los fenómenos de transporte de los metales pesados en base a los estudios de espectroscopia de absorción atómica (27) y microscopia de barrido electrónico (SEM) (28)

La contaminación por metales pesados se difunde en áreas extensas (29) y de difícil acceso (30), por ende, es difícil transportar estos suelos contaminados a un centro de tratamiento ambiental "ex-situ" (31), viéndose reflejados problemas como: transporte, tiempo, costos y riesgos etc. (32).

Por esta razón se presenta al tratamiento electrocinético como una alternativa viable para tratar suelos contaminados (33), afectados por las actividades hidrocarburíferas (34), mediante la aplicación de una diferencia de potencial y la inyección de soluciones humectantes en el suelo (35), movilizando los iones presentes hacia los electrodos (36), así aislando el contaminante para su recuperación, tratamiento y/o disposición final (37).

Esta técnica se hace atractiva por ser "in-situ", amigable con el medio ambiente (38), y principalmente no involucra costos excesivos para su implementación (39) y, aplicación frente a las otras tecnologías de tratamiento (40).

\section{MATERIALES Y MÉTODOS}

- $\quad$ El área de influencia se encuentra ubicado en las coordenadas, Este: $291149.11 \mathrm{mE}$, Norte:9963334.93 mS, z18m; a una altitud de270 m.s.n.m. Con una longitud: $-76.877804^{\circ}$ y latitud: $-0.333529^{\circ}$.

El área muestreada se inició a $100 \mathrm{~m}$ del centro poblado sector la Parker vía Sacha - Coca. Con la finalidad de obtener muestras compuestas de suelo, se realizó un muestreo aleatorio simple a los alrededores de la estación de operaciones central Petroamazonas (PAM), con una longitud de muestreo de $3386 \mathrm{~m}$; 


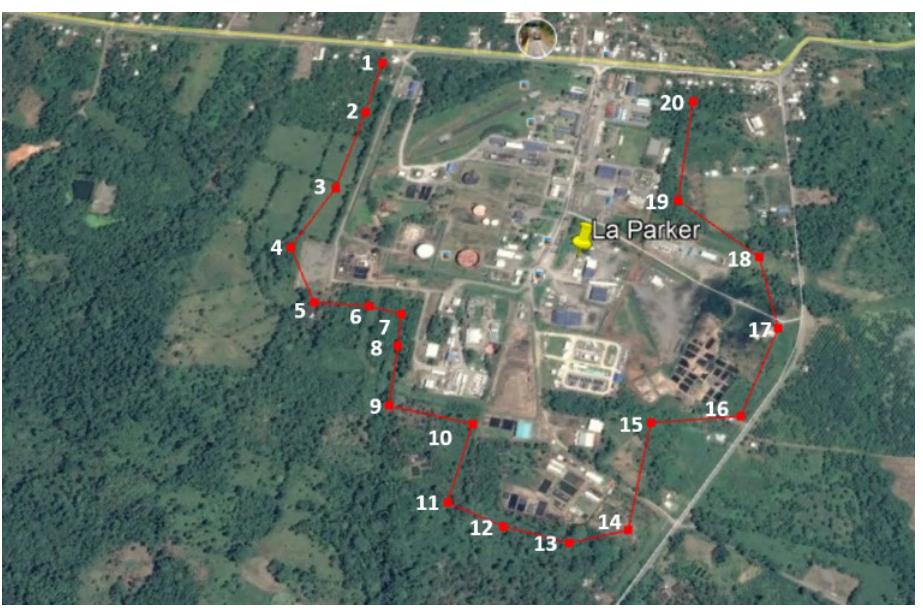

Gráfico 1. Zona de Estudio en el cantón Joya de los Sachas, sector La Parker (Yaulema et al, 2018)

Fuente: Google Earth, 2018.

Del área delimitada se observan 20 puntos equivalente al número de submuestras de $1 \mathrm{~kg}$ cada uno, se realizó un cuarteo donde se tomó una muestra compuesta homogénea, colocadas en una funda ziploc y almacenadas en un termo a baja temperatura evitando la volatilización, para luego ser analizadas en el Laboratorio de Suelos de la Facultad de Ciencias de la ESPOCH; previamente se envió una muestra de $100 \mathrm{~g}$ al Laboratorio de Análisis y Evaluación Ambiental (AQLAB en Orellana, Ecuador) para determinar la concentración de $\mathrm{Cd}$ y $\mathrm{Ni}$ en la muestra.

En vista que las concentraciones $\mathrm{Cd}$ y $\mathrm{Ni}$ originales de las muestras tomadas en el lugar de estudio no tuvieron la concentración suficiente como para llevar a cabo los experimentos de electrorremediación, se procedió a adicionar $\mathrm{Cd}$ en el suelo contaminado debido a que, las concentraciones estaban debajo de los límites permisibles para llevar a cabo esta investigación.

\section{Caracterización física, fisicoquímica y mineralógica de la muestra de suelo.}

Los principales parámetros físicos y fisco-químicos del suelo que se tomaron en cuenta y se analizaron por triplicado fueron: tamaño de la partícula, densidad aparente, densidad real, porosidad, color, porcentaje de humedad, textura, capacidad de intercambio catiónico (C.I.C) bajo la norma PEE/SFA/14, pH con norma EPA 9045D y conductividad eléctrica (CE) con la norma EPA 9050A. Para el análisis estadístico, se empleó la prueba de Tukey.

\section{Determinación del tamaño de partícula}

Se tomaron 500 g de muestra de suelo seco y homogéneo contaminado con Cd y Ni. Se dispuso de un arreglo de tamices formado por el tamiz \#01 (475 mm) y el tamiz \#11 (53 $\mu \mathrm{m})$, sobre este se colocó el material, y se agitó durante $15 \mathrm{~min}$; se retiró el arreglo de tamices y se cuantificó el peso del material retenido en cada uno de ellos. El material retenido en el fondo se colocó sobre un segundo arreglo de tamices para luego repetir el proceso anteriormente descrito con el propósito de diferenciar el área específica de reacción.

\section{Determinación de la densidad aparen- te:}

Se dispuso de una probeta de $50 \mathrm{~mL}$ previamente tarada en la balanza analítica BOECO BAS 31 plus y se colocó un volumen de $20 \mathrm{~mL}$ ¿de qué?; se registró el peso de 4,84g ocupado por el material para establecer la relación entre la masa $(22,16$ g) y el volumen ocupado por la misma. Para la determinación de la densidad real, se utilizó un picnómetro ISOLAB (27).

\section{Determinación de la acidez del suelo ( $\mathrm{pH}$ ) y la conductividad eléctrica (CE)}

Se pesaron $10 \mathrm{~g}$ de suelo seco, tamizado y homogenizado y se añadieron a un vaso de precipitación de $100 \mathrm{~mL}$, junto con $25 \mathrm{~mL}$ de agua destilada. La suspensión se agitó durante $30 \mathrm{~min}$ con un agitador magnético, se dejó en reposo durante $1 \mathrm{~h}$ para favorecer su decantación y se determinó el pH y la CE de la muestra.

Caracterización mineralógica y elemental de la muestra de suelo contaminada con metales pesados.

Para estos análisis se envió $100 \mathrm{~g}$ de muestra compuesta, seca y homogenizada al Departamento de Metalurgia extractiva de la Escuela Politécnica Nacional (EPN), en el cual se determinó: compuestos cristalinos, empleando el difractómetro D8 ADVANCE y el pro- 
grama Diffrac plus (EVA Y TOPAS) para cualificación y semi-cuantificación; así como también un análisis semi-cuantitativo por Microscopia Electrónica de Barrido, empleando el microscopio TESCAN-VEGA, con micro analizador de rayos $\mathrm{X}$ Bruker (41).

\section{Otros parámetros analizados.}

Parámetros como el color, humedad, textura, granulometría y la concentración de metales pesados ( $\mathrm{Cd}, \mathrm{Ni})$ se determinaron en el Laboratorio de Análisis y Evaluación Ambiental (AQLAB) acreditado por el Servicio de Acreditación Ecuatoriana $\mathrm{N}^{\circ}$ OAE LE C 14-009; y el parámetro capacidad de intercambio catiónico (C.I.C) se determinó en AGROCALIDAD.

\section{Diseño e implementación del sistema experimental.}

El equipo de electrorremediación se rediseñó, determinando el tiempo y las distancias optimas entre los electrodos, definiendo que para un sistema de electrorremediación a escala laboratorio se debe trabajar a una distancia de $15 \mathrm{~cm}$ entre los electrodos anódico y catódico, en un tiempo superior a las $24 \mathrm{~h}$.

El material de la cubeta donde se remedió el suelo contaminado con metales pesados es de vidrio templado de 10 $\mathrm{mm}$, cuyas dimensiones son $25 \mathrm{~cm}$ de largo, $10 \mathrm{~cm}$ de ancho y $13 \mathrm{~cm}$ de alto; la distancia entre electrodo es de $15 \mathrm{~cm}$ con pozos de $2,5 \mathrm{~cm}$ cada uno. El compartimento medio, puede albergar aproximadamente $1200 \mathrm{~cm} 3$ de muestra de suelo a tratar.

Los electrodos utilizados son de grafito, ya que es un material neutro, con una alta capacidad conductora, y muy accesible económicamente. Con las siguientes dimensiones $10 \times 8 \times 1,5 \mathrm{~cm}$ de alto, ancho y espesor respectivamente. Además, se contó con una fuente de poder de voltaje y amperaje regulable de 0 a $60 \mathrm{~V}$ y 0 a $5 \mathrm{~A}$, con dos conductores eléctricos dotados con pinzas (roja carga positiva, negra carga negativa).

\section{Preparación de los electrolitos.}

Se calculó la cantidad de ácido acético $(\mathrm{CH} 3 \mathrm{COOH} 0,1$ M) y de ácido fosfórico (H3PO4 0,1 M) necesaria para el experimento. La cantidad de ácido acético requerida, con la ayuda de una pipeta se tomó $5,7 \mathrm{~mL}$ de ácido acético glacial con concentración $99,75 \%$, y se aforó a 1000 $\mathrm{mL}$ en agua destilada; el mismo proceso se usó para preparar el ácido fosfórico, se tomó 6,1 mL de H3PO4, con concentración del 96\% y se aforó a 1000 mL, (Nunca se agrega agua al acido, si no acido al agua para evitar una reacción explosiva) (42)

\section{Preparación del agente humectante.}

La solución humectante utilizada fue sulfato de hierro 0,1 $\mathrm{M}$ (FeSO4). Se saturó la muestra de suelo con $185 \mathrm{~mL}$ del agente humectante (FeSO4 0,1 M), motivo por el cual se pesó 28,07 g de FeSO4, y diluyó a $1000 \mathrm{~mL}$, agitando manualmente hasta que el sólido se disolvió.

\section{Condiciones iniciales de operación.}

La duración de cada experimento fue de 48 h, sustituyendo las soluciones electrolíticas cada $4 \mathrm{~h}$. En la operación experimental se trabajó con gradientes de voltaje de 25 $\mathrm{V}$ a $55 \mathrm{~V}$ con intervalos de $10 \mathrm{~V}$, el paso de corriente en el proceso se monitoreó mediante un multímetro, en periodos de $2 \mathrm{~h}$.

En el pozo anódico se colocó una solución de $95 \mathrm{~mL}$ de (CH3COOH 0,1 M), y en el pozo catódico $95 \mathrm{~mL}$ de (H3PO4 0,1 M).

Para la humectación de 600 g suelo contaminado, la muestra se saturó con $185 \mathrm{~mL}$ de (FeSO4 0,1 M), en un depósito, mezclándola con movimientos homogéneos circulares durante 5 minutos. Luego se dejó reposar durante $5 \mathrm{~h}$ antes de iniciar el proceso de electrorremediación.

Durante el proceso, se llevó a cabo un seguimiento de las variaciones de $\mathrm{pH}$ en los pozos anódico y catódico utilizando el pH-metro WTW pH 3110 en intervalos regulares. 


\section{RESULTADOS Y DISCUSIÓN}

Caracterización físicoquímica de la muestra de suelo.

\begin{tabular}{lccc}
\hline \multicolumn{1}{c}{ Parámetro analizado } & Referencia & Resultado & Unidad \\
\hline Capacidad de intercambio catiónico (C.I.C.) & PEE/SFA/14 & 13,86 & $\mathrm{cmol} / \mathrm{kg}$ \\
pH & EPA 9045D & 4,61 & $\sim$ \\
Conductividad eléctrica (C.E.) & EPA 9050A & 195,70 & $\mu \mathrm{S} / \mathrm{cm}$ \\
$\begin{array}{l}\text { Tabla 1. Caracterización físicoquímica inicial de la muestra de suelo (Yaulema et al, 2018) } \\
\text { Fuente: Laboratorios FC-ESPOCH, AGROCALIDAD. }\end{array}$ &
\end{tabular}

Caracterización mineralógica de la muestra de suelo.

\begin{tabular}{|c|c|c|}
\hline Mineral & Formula & Contenido (\%) \\
\hline Cuarzo & $\mathrm{SiO}_{2}$ & 54 \\
\hline Caolinita & $\mathrm{Al}_{2}\left(\mathrm{Si}_{2} \mathrm{O}_{5}\right)(\mathrm{OH})_{4}$ & 24 \\
\hline Muscovita & $\mathrm{KAl}_{2}\left(\mathrm{AlSi}_{3} \mathrm{O}_{10}\right)(\mathrm{OH})_{2}$ & 8 \\
\hline Vermiculita & $(\mathrm{Mg}, \mathrm{Fe}, \mathrm{Al})_{6}(\mathrm{OH})_{4}(\mathrm{Al}, \mathrm{Si})_{8} \mathrm{O}_{20}{ }^{*} 4 \mathrm{H}_{2} \mathrm{O}$ & 3 \\
\hline Nontronita & $\mathrm{Na}_{0,3}\left(\mathrm{Fe}^{3+}\right)_{2}(\mathrm{Si}, \mathrm{Al})_{4} \mathrm{O}_{10}(\mathrm{OH})_{2}^{*} \mathrm{nH}_{2} \mathrm{O}$ & 3 \\
\hline Clinocloro & $(\mathrm{Mg}, \mathrm{Fe})_{5} \mathrm{Al}(\mathrm{Si}, \mathrm{Al})_{4} \mathrm{O}_{10}(\mathrm{OH})_{8}$ & 2 \\
\hline Grupo plagioclasas (albita, andesina, anortita) & $(\mathrm{Na}, \mathrm{Ca}) \mathrm{Al}(\mathrm{Si}, \mathrm{Al}) \mathrm{Si}_{2} \mathrm{O}_{8}$ & 2 \\
\hline Flogopita & $\mathrm{KMg}_{3}(\mathrm{~F}, \mathrm{OH})_{2} \mathrm{AlSi}_{3} \mathrm{O}_{10}$ & 2 \\
\hline Dolomita & $\mathrm{CaMg}\left(\mathrm{CO}_{3}\right)_{2}$ & 1 \\
\hline $\begin{array}{l}\text { Calcita } \\
\text { Tabla 2. Composición mineralógica inicial de la muestra de sue }\end{array}$ & $\frac{\mathrm{CaCO}_{3}}{\text { o. (Yaulema et al, 2018) }}$ & 1 \\
\hline
\end{tabular}

Identificación de los potenciales óptimos de remediación.

\begin{tabular}{|c|c|c|c|c|c|c|c|c|}
\hline \multirow{2}{*}{$\begin{array}{l}\text { Trata- } \\
\text { miento }\end{array}$} & \multicolumn{2}{|c|}{$[\mathrm{Cd}] \mathrm{mg} / \mathrm{kg}$} & \multicolumn{2}{|c|}{ [Ni] mg/kg } & \multirow{2}{*}{$\begin{array}{c}\text { Voltaje } \\
(\mathrm{V})\end{array}$} & \multirow{2}{*}{$\begin{array}{c}\text { Amperaje } \\
(\mathrm{mA})\end{array}$} & \multirow{2}{*}{$\begin{array}{c}\text { Área de los } \\
\text { electrodos }\left(\mathrm{cm}^{2}\right)\end{array}$} & \multirow{2}{*}{$\begin{array}{l}\text { Densidad de corriente } \\
\qquad\left(\mathrm{mA} / \mathrm{cm}^{2}\right)\end{array}$} \\
\hline & Inicial & Final & Inicial & Final & & & & \\
\hline $\mathrm{T} 1$ & 406 & 151,05 & 250 & 84,10 & 25 & 90 & 60 & 1,50 \\
\hline $\mathrm{T} 2$ & 406 & 84,80 & 250 & 61,55 & 35 & 180 & 60 & 3,00 \\
\hline T3 & 406 & 25,55 & 250 & 37,20 & 45 & 240 & 60 & 4,00 \\
\hline $\mathrm{T} 4$ & 406 & 39,70 & 250 & 45,65 & 55 & 280 & 60 & 4,67 \\
\hline
\end{tabular}

Prueba estadística

\begin{tabular}{|c|c|c|c|c|c|c|c|c|c|}
\hline \multicolumn{10}{|c|}{ HSD Tukey $^{\mathrm{a}}$} \\
\hline \multicolumn{10}{|c|}{ Subconjunto para alfa $=\mathbf{0 . 0 5}$} \\
\hline \multirow{2}{*}{$\begin{array}{c}\text { Voltaje apli- } \\
\text { cado }\end{array}$} & \multirow{2}{*}{$\mathbf{N}$} & \multicolumn{4}{|c|}{$[\mathrm{mg} / \mathrm{kg}] \mathrm{Cd}$} & \multicolumn{4}{|c|}{$[\mathrm{mg} / \mathrm{kg}] \mathrm{Ni}$} \\
\hline & & 1 & 2 & 3 & 4 & 1 & 2 & 3 & 4 \\
\hline 45 & 3 & 25,55 & & & & 37,20 & & & \\
\hline 55 & 3 & & 39,70 & & & & 45,65 & & \\
\hline 35 & 3 & & & 84,80 & & & & 61,55 & \\
\hline 25 & 3 & & & & 151,05 & & & & 84,10 \\
\hline Sig. & & 1,00 & 1,00 & 1,00 & 1,00 & 1,00 & 1,00 & 1,00 & 1,00 \\
\hline \multicolumn{10}{|c|}{ Se visualizan las medias para los grupos en los subconjuntos homogéneos. } \\
\hline
\end{tabular}


Análisis de los fenómenos de transporte mediante estudios de espectroscopia de absorción atómica y SEM.

\begin{tabular}{lll}
\hline \multicolumn{1}{c}{ Parámetros } & \multicolumn{1}{c}{ Cadmio } & \multicolumn{1}{c}{ Níquel } \\
\hline Agente humectante empleado & FeSO $_{4} 0,1 \mathrm{M}$ & FeSO4 0,1 M \\
Concentración inicial de la muestra de suelo sin tratar $(\mathrm{mg} / \mathrm{kg})$ & $406,00 \pm 1,25$ & $250,00 \pm 2,20$ \\
Concentración promedio de la muestra de suelo tratado a 45 V, (mg/kg) & 26,80 & 39,40 \\
Observación & Cambio significativo & Cambio significativo \\
Tabla 5. Resultados de migración iónica de Cd y Ni después del tratamiento electrocinético (Yaulema et al, 2018) &
\end{tabular}

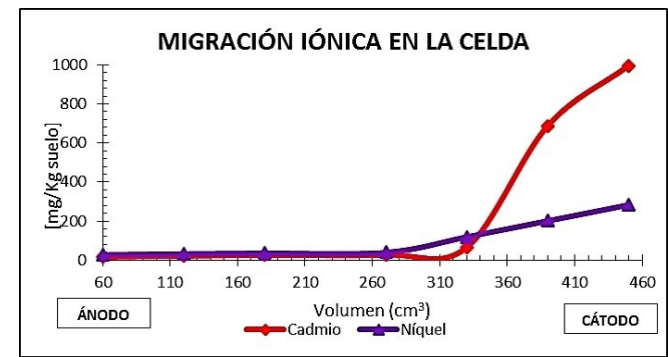

Gráfico 2 Perfil de concentración del Cd y Ni a ciertos

volúmenes, durante 48 h, a $45 \mathrm{~V}$ (Yaulema et al, 2018)

En relación al Gráfico 2, se debe estudiar la cinética y dinámica del proceso con una mayor cantidad de experimentos, variando las condiciones.

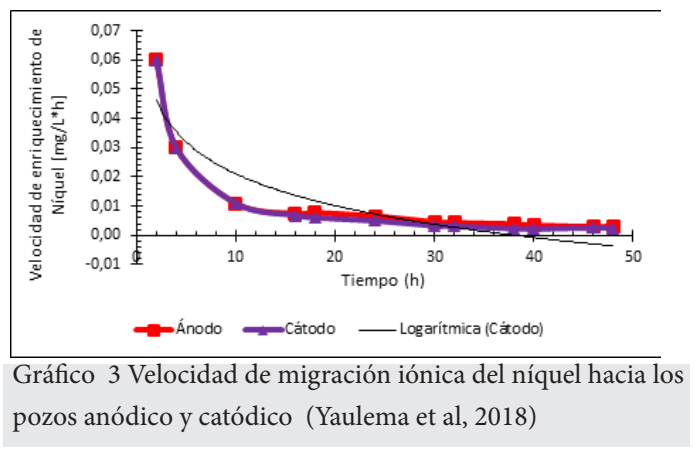

La curva mostrada en el Gráfico 3, puede describirse con una función logarítmica, de la siguiente manera $\mathrm{y}=-0,016 \ln (\mathrm{x})+0,0574$

Balance de masas de cadmio y níquel presente en la celda y los pozos.

\begin{tabular}{|c|c|c|c|}
\hline Parámetro & & Concentración (ppm) & Masa $(\mathrm{mg})$ \\
\hline \multirow[t]{2}{*}{ Inicial } & $\mathrm{cm}$ & 250,00 & 150,00 \\
\hline & $15-13$ & 283,08 & \\
\hline \multirow[t]{2}{*}{ Región más concentrada } & $13-11$ & 201,02 & 144,75 \\
\hline & $11-9$ & 119,00 & \\
\hline \multirow[t]{2}{*}{ Región central } & $9-6$ & 39,40 & 4,74 \\
\hline & $6-4$ & 35,07 & \\
\hline \multirow[t]{2}{*}{ Región menos concentrada } & $4-2$ & 32,01 & 22,63 \\
\hline & $2-0$ & 27,20 & \\
\hline \multicolumn{2}{|l|}{ Pozo anódico } & 1,63 & 0,15 \\
\hline \multicolumn{2}{|l|}{ Pozo catódico } & 1,31 & 0,12 \\
\hline \multicolumn{2}{|l|}{ Total solido } & $\sim$ & 172,12 \\
\hline \multicolumn{2}{|l|}{ Total solución } & $\sim$ & 0,27 \\
\hline
\end{tabular}


A continuación, se muestran los valores en concentración y masa del cadmio, antes y después del tratamiento electrocinético:

\begin{tabular}{lccc}
\hline \multicolumn{1}{c}{ Parámetro } & & Concentración & Masa \\
\hline \multicolumn{1}{c}{ Inicial } & $\mathbf{c m}$ & $406,00 \mathrm{ppm}$ & $243,60 \mathrm{mg}$ \\
\hline \multirow{2}{*}{ Región más concentrada } & $15-13$ & $996,40 \mathrm{ppm}$ & \\
& $13-11$ & $688,50 \mathrm{ppm}$ & $420,24 \mathrm{mg}$ \\
& $11-9$ & $66,09 \mathrm{ppm}$ & \\
\hline Región central & $9-6$ & $26,80 \mathrm{ppm}$ & $3,22 \mathrm{mg}$ \\
\hline & $6-4$ & $25,40 \mathrm{ppm}$ & \\
Región menos concentrada & $4-2$ & $21,02 \mathrm{ppm}$ & $14,99 \mathrm{mg}$ \\
& $2-0$ & $16,03 \mathrm{ppm}$ & \\
\hline Total solido & $\sim$ & $438,45 \mathrm{mg}$ \\
\hline $\begin{array}{l}\text { Tabla 7. Balance del cadmio durante el proceso de electrorremediación (Yaulema et } \\
\text { al, 2018) }\end{array}$ & & \\
Fuente: Laboratorios DEMEX-EPN. &
\end{tabular}

\section{Análisis de microscopía de barrido electrónico.}

\begin{tabular}{lcc}
\hline Elemento & $\begin{array}{c}\text { Contenido elemental antes del } \\
\text { tratamiento (\%) }\end{array}$ & $\begin{array}{c}\text { Contenido elemental después del } \\
\text { tratamiento (\%) }\end{array}$ \\
\hline $\mathbf{S i}$ & 33,1 & 14,38 \\
$\mathrm{Al}$ & 26,7 & 8,05 \\
$\mathrm{Fe}$ & 5,96 & 3,20 \\
$\mathbf{K}$ & 4,5 & 0,39 \\
$\mathbf{M g}$ & 2,72 & 0,33 \\
\hline Tabla 8. Caracterización química elemental final de la muestra de suelo (Yaulema et \\
al, 2018) \\
Fuente: Laboratorios DEMEX-EPN.
\end{tabular}

\section{DISCUSIÓN}

En la Tabla 1, se observa que la baja capacidad de intercambio catiónico, se debe al suelo franco limoso, por lo tanto, esto afecta el movimiento y retención de cationes y a la capacidad tampón del suelo (2). Además, la baja conductividad eléctrica representa un suelo no salino, esto se corrobora con las bajas concentraciones de sales, reflejadas en la C.I.C. El pH, en base al departamento de Agricultura de los Estados Unidos (USDA por sus siglas en inglés) es un suelo fuertemente acido, por la presencia de aluminosilicatos (43). Generalmente, los metales son móviles a pH bajo, en forma de especies iónicas libres o como órgano-metales solubles; al $\mathrm{pH}$ alcalino forman carbonatos o fosfatos minerales insolubles (36).

Los resultados obtenidos durante el análisis de la muestra de suelo contaminada con Cd y Ni (Tabla 3), fueron comparados con la Tabla 6 denominada "límites permisibles para la identificación y remediación de suelos contaminados en todas las fases de la industria hidrocarburífera...", del reglamento ambiental de actividades hidrocarburiferas (RAOHE 1215), los valores fueron mayores a los límites máximos permisibles, donde el cadmio tiene una concentración inicial de $406 \mathrm{mg} / \mathrm{kg}$ de suelo, y el níquel $250 \mathrm{mg} / \mathrm{kg}$ de suelo, estos valores determinan al suelo como altamente contaminado.

Suelos con altas concentraciones de metales pesados como $\mathrm{Cd}$ y Ni en limites no permisibles hacen imposible ser depositados directamente al medio ambiente, la presencia de estos contaminantes en el medio se ve relacionado con problemas de bioacumulación, persistencia, problemas desfavorables para la vida, etc.

En la Tabla 2, se evidenció mayoritariamente la presencia de minerales primarios como los silicatos, aunque también se presentaron óxidos e hidróxidos, fluoruros y carbonatos, el alto porcentaje de cuarzo y caolinita se debe a la presencia de silicio y aluminio, responsables de la acidez de los suelos.

El silicato más resistente a la alteración es el cuarzo y es el más abundante en las arenas de los suelos. En las caolinitas, el silicio no se sustituye nunca, su partícula elemental es eléctricamente neutra, su C.I.C es muy baja (1-10 $\mathrm{cmol}(+) / \mathrm{kg})$, lo que explica la baja fertilidad de los suelos ricos en caolinita. La vermiculita no es muy frecuente. Son arcillas de tipo intermedio entre las cloritas y las micas, son expandibles, lo que permite la entrada de agua y cationes que sustituyen al $\mathrm{Mg} 2+$.

La Tabla 3, muestra que cada experimento se realizó en un tiempo continuo de 48 h, se usaron soluciones electrolíticas para atraer la migración iónica de los contaminantes hacia los pozos receptores. En el pozo anódico se utilizó ácido acético, y en el pozo catódico se utilizó ácido fosfórico, humectando la muestra durante $5 \mathrm{~h}$ con sulfato de hierro, cabe mencionar además que todas las soluciones utilizadas tuvieron una concentración 0,1 molar. Siendo el tercer tratamiento (T3) con condiciones de $45 \mathrm{~V}$ y $240 \mathrm{~mA}$, el que representa una mayor 
eficiencia de remoción del 93,7\% para el caso del cadmio; y para el níquel $85,1 \%$. Existiendo además una diferencia entre la aplicación de diferentes voltajes. La diferencia con los tratamientos T1, T2 y T4, es básicamente la diferencia de voltajes: $25 \mathrm{~V}, 35 \mathrm{~V}$ y $45 \mathrm{~V}$ respectivamente, y por tanto la variación de su amperaje y densidad de corriente.

La prueba estadística de Tukey que se muestra en la Tabla 4, aplicada al tratamiento de níquel demostró que, en los cuatro tratamientos de electrorremediación el voltaje óptimo es el de $45 \mathrm{~V}$, en el cual se disminuyó con mayor eficiencia el contaminante presente en la muestra. Como segundo tratamiento más eficiente se presentó al experimento cuatro a 55 V.

La determinación del desplazamiento iónico está basada en el experimento tres (T3), donde existe una diferencia con la concentración promedio del suelo tratado; es decir se encuentra fuera del rango de incertidumbre. En el caso del níquel la concentración final del suelo tratado se encuentra fuera del rango de incertidumbre con respecto a la concentración de suelo sin tratar. Mediante esta técnica de discriminación iónica, se definió que existió un cambio significativo en las concentraciones de cadmio y níquel después de aplicar el tratamiento electrocinético; lo cual demuestra una alta eficiencia en la aplicación de esta tecnología.

En la Tabla 5, se observa que la mayor concentración de cadmio reportada después del tratamiento electrocinético en la muestra de $996,40 \mathrm{mg} / \mathrm{kg}$ de suelo, en un volumen de $60 \mathrm{~cm} 3$, comprendidos entre los centímetros 13 y 15 cercanos al cátodo; mientras que en las zonas intermedias comprendidas entre los centímetros 6 y 9 , en un volumen de $90 \mathrm{~cm} 3$ . Se obtuvo una disminución del metal, que alcanzó un promedio de 26,80 ppm, además, en los $2 \mathrm{~cm}$ cercanos al ánodo se evidencia una concentración baja de
16,03 ppm, en estas regiones la concentración de cadmio es menor. Esto se debe a que la mayoría de los metales se encuentran en forma de cationes, los mismos que migraron y se acumularon en las zonas cercanas al cátodo (polo negativo).

Con relación al enriquecimiento de cadmio y níquel en los pozos anódico y catódico que se muestra en los gráficos 2 y 3. Se demostró que la migración iónica de los metales de interés se encontró favorecida hacia el cátodo, además, se presentan los perfiles de velocidad y la concentración acumulada del níquel en ambos pozos. Cabe mencionar, que la migración del cadmio se despreció, ya que mediante los análisis de absorción atómica los resultados reportados fueron menores al rango de detección del equipo $(<0,05) \mathrm{ppm}$.

Durante el proceso, la velocidad de enriquecimiento de níquel en ambos pozos fue disminuyendo progresivamente, debido que las soluciones tienden a saturarse, por ello se sustituyeron periódicamente las mismas. Esto, favoreció una migración dirigida de los iones metálicos, durante las primeras $10 \mathrm{~h}$ de tratamiento, en la que existió una movilidad significativa en ambos pozos, y pasadas las 30 h se evidenció una cierta estabilización con un valor promedio $0,004 \mathrm{mgNi} / \mathrm{L} \cdot h$ en el ánodo, y en el cátodo una velocidad de enriquecimiento menor $0,003 \mathrm{mgNi} / \mathrm{L} \cdot \mathrm{h}$. La presencia del contaminante en ambos pozos demuestra la existencia de níquel en formas aniónicas y catiónicas.

En la Tabla 6, debido a la baja migración iónica en los pozos, se debe saber la concentración total de níquel en el sistema. La región de mayor acumulación fue la cercana al cátodo en una distancia comprendida entre 9 y $15 \mathrm{~cm}$; mientras que en la zona céntrica comprendida entre los 6 y $9 \mathrm{~cm}$ en un volumen de $90 \mathrm{~cm} 3$ se obtuvo una concentración baja en comparación a la inicial.

Entre los 0 y $6 \mathrm{~cm}$ contiguos al ánodo, la masa es menor en comparación con la masa acumulada en la zona aledaña al cátodo, esto se debe a que la mayoría del níquel se encuentra en forma de cationes y es atraída por el campo catódico. La masa acumulada de níquel presente en los pozos es relativamente baja, en el pozo anódico existe una mayor acumulación de níquel con respecto al pozo catódico con valores de 0,15 y $0,12 \mathrm{mg}$, respectivamente.

En la Tabla 7, se demuestra que al igual que el níquel, el cadmio tiene la misma tendencia de desplazamiento iónico en la celda, presentando una baja o nula migración iónica en los pozos, por ello se debe saber la concentra- 
ción total de cadmio en el sistema, se analizó diferentes volúmenes de muestra en la celda. La región de mayor acumulación fue la cercana al cátodo en una distancia comprendida entre 9 y $15 \mathrm{~cm}$, mientras que en la zona céntrica comprendida entre los 6 y $9 \mathrm{~cm}$ se obtuvo una concentración baja en comparación a la inicial.

Entre los 0 y $6 \mathrm{~cm}$ contiguos al ánodo, la masa de cadmio, es menor en comparación con la masa acumulada en la zona aledaña al cátodo, esto se debe a que la mayoría de iones de cadmio se encuentran en forma catiónica y son atraídos por el campo catódico. La masa acumulada en los pozos anódico y catódico fue despreciable debido a valores reportados menores a $<0,05 \mathrm{ppm}$, rango no detectable por el equipo de absorción atómica.

Los análisis realizados por la técnica microscopia de barrido electrónico se efectuaron después del tratamiento electrocinético (Tabla 8). En la muestra que se trabajó a $45 \mathrm{~V}$ en un tiempo de remediación de $48 \mathrm{~h}$, la zona analizada comprende desde 10 a $15 \mathrm{~cm}$ de la muestra, desde el ánodo hacia el cátodo, en un volumen de $150 \mathrm{~cm} 3$, obteniéndose así, una disminución de los iones iniciales presentes en la muestra sin tratamiento. El silicio presentó una disminución del 43,44\%, el aluminio 30,15\%, el hierro $53,69 \%$, potasio $8,67 \%$ y el magnesio un $12,13 \%$. Esto se debe al desplazamiento de los iones hacia los electrodos siendo atraídos por el campo eléctrico generado. En el Gráfico 4, se muestran microfotografías capturadas por el microscopio donde evidencia una mayor acumulación y saturación de las partículas del suelo, además se evidencia la reducción del tamaño de partícula.

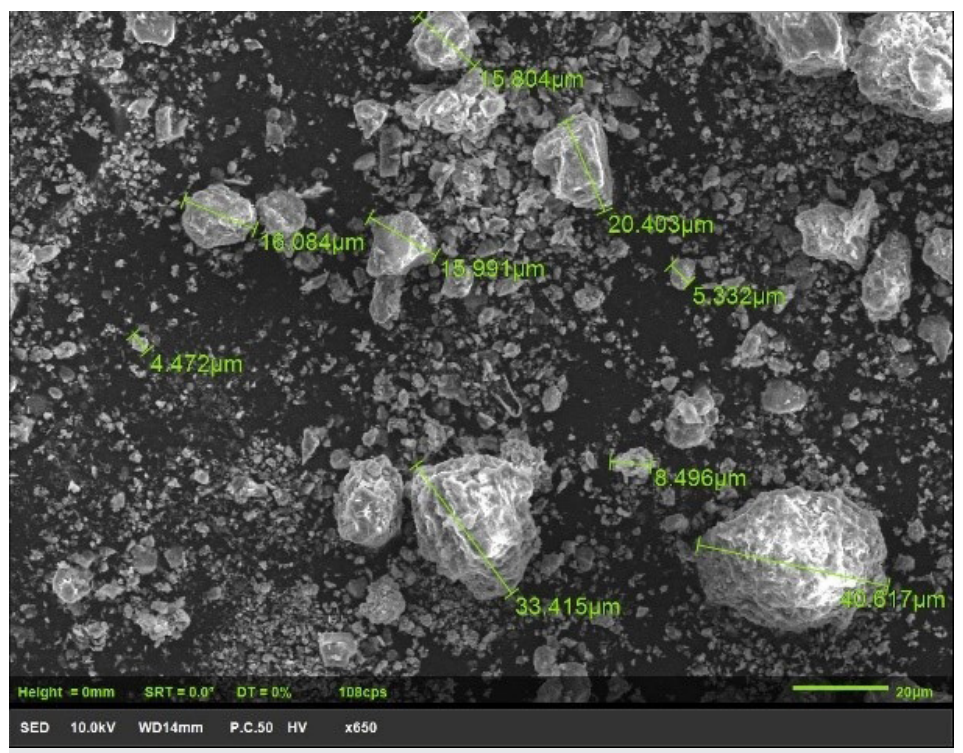

Gráfico 4 Muestra tratada, obtenida por microscopía de barrido electrónico (Yaulema et al, 2018)

\section{CONCLUSIONES}

La electrorremediación es una técnica poco estudiada en nuestro país, tiene un gran potencial para tratar diversos tipos de contaminantes como compuestos orgánicos e inorgánicos, se seleccionó el potencial óptimo de remediación, durante un periodo de $48 \mathrm{~h}$, mediante la aplicación de soluciones electrolíticas e humectantes para movilizar los iones metálicos presentes en la muestra que permitieron recuperar el suelo contaminado.

La concentración inicial de metales pesados cadmio y níquel en la muestra, superan los límites máximos permisibles, establecidos por la Tabla 6 del RAOHE 1215 para uso industrial, con una concentración de cadmio 406 ppm, y el níquel 250 ppm en contraste con la norma que sugiere 10 y $100 \mathrm{ppm}$ respectivamente. Se debe mencionar, además, que la aplicación de procesos electrocinéticos es más efectiva en suelos arcillosos debido a su carga superficial negativa.

En la identificación de los potenciales óptimos de remediación de la muestra de suelo contaminada con metales pesados cadmio y níquel, se seleccionó al tratamiento (T3) con un voltaje constante de $45 \mathrm{~V}$ a una intensidad de corriente inicial de $0,24 \mathrm{~A}$.

La concentración final de $\mathrm{Cd}$ fue de 25,55 ppm y una eficiencia máxima de remoción de 93,71\%, mientras que para el níquel una concentración final de $37,20 \mathrm{ppm}$ con una eficiencia de remoción de 85,12\%, permitiendo cumplir con la normativa aplicable para actividades hidrocarburíferas RAOHE 1215.

Existe una migración iónica dirigida hacia el campo catódico, esquematizándose una mayor acumulación de los iones metálicos en una distancia comprendida entre los 9 y $15 \mathrm{~cm}$ contiguos al cátodo, 
mientras que, en el otro extremo cercano al ánodo, la concentración de estos metales es relativamente baja.

La migración iónica de $\mathrm{Ni}$ en los pozos tuvo una acumulación mínima de los iones metálicos, sin embargo, presentó una mayor cantidad de migración al pozo anódico, que, al catódico.

Esto se debe a la presencia de níquel en formas anicónicas, el desplazamiento de cadmio fue despreciable no existió una acumulación significativa de este metal en los pozos.

\section{$\mathrm{R}$}

1. Jaramillo J. Introducción a la Ciencia del Suelo Suelos y Ambiente. 2002.

2. López VL, Rubén Remediación electrocinética de suelos de baja permeabilidad contaminados con compuestos orgánicos hidrófobos Universidad de Castilla la Mancha, Ciudad Real, España. 2013;1(12):34-44.

3. Oviedo RA, B, . Contaminación por metales pesados en el sur del Ecuador asociada a la actividad minera. . Bionatura. 2017;2(4):437-41.

4. ECOLOGÍA IND. Composición y propiedades del suelo. Ecología Nacional. 2018:22-32.

5. Escobar AO, Segundo. Electrorremediación en función de la densidad de corriente y la concentración de ácido acético, como alternativa para la disminución de plomo en relaves mineros - mina paredones. niversidad Privada del Norte Cajamarca. 2016:105.

6. Mosquera SM. Evaluación de procesos no convencionales de remediación de relaves sulfurados de minería contaminados con cobre, zinc y plomo. Escuela Politécnica Nacional, Quito. 2014:1.219. 7. Aragón CDlT, Ernesto; Guevara, Alicia., . Diseño a escalas laboratorio y piloto de un sistema de remediación electrocinética de suelos contaminados con metales pesados. Revista EPN. 2014;vol. 34:9-15.

8. ARPEL. Tratamiento y eliminación de desperdicios de perforación de exploración y producción. Alconsult International Ltd Montevideo, Uruguay 2013:116.

9. Reglamento Ambiental de Actividades Hidrocarburíferas 1215 (1998).

10. Texto Unificado de Legislación secundaria del Ministerio del Ambiente: Norma de Calidad Ambiental del Recurso Suelo y Criterios de Remediación para suelos contaminados. , (2015).

11. Acuerdo No. 061 Reforma del Libro VI del Texto Unificado de Legislación Secundaria. , (2015).

12. Martínez L. Lo que usted debe saber del níquel Muy interesnte digital, 2012, Austin, Texs no 800. 2012;1:2.

13. Miranda Cabrera MC. Eliminación de metales pesados en el agua de reinyección del campo libertador oeste utilizando quitosán Escuela Superior Politécnica de Chimborazo, Riobamba, Ecuador. 2006(1):209.

14. Moreno P. Disminución de la concentración de cadmio en el suelo con la técnica de electrorremediación en una celda construida en el laboratorio Universidad Autónoma Agraria Antonio Narro Unidad Laguna, Torreón, México 2017:72.

15. Nordberg Gea. Metales: Propiedades químicas y toxicidad Metales en el suelo. 2001;3ra ed. Ginebra.

16. Granda JF. Evaluación de la técnica de electrorremediación de suelos contaminados por metales pesados. Universidad Técnica Particular de Loja. 2011:79.

17. Gilbon M. Electroremediación de suelos contaminados con hidrocarburos. . Tesis de Licenciatura en Química. : Universidad Autónoma Metropolitana, ; 2001.

18. Molina Díaz GE, Palestina Caliz V, Piedras Pérez JA. Recuperación electrocinética de hidrocarburos sorbidos en suelos contaminados. Universidad Autónoma Metropolitana: Universidad Autónoma Metropolitana; 2004.

19. Hamed J. AYByGRJ. $\mathrm{Pb}$ (II) removal from kaolinite by electrokinetics.

. J Geotech Eng. 1991:117, 241-71. 
20. García H, Vargas R, Reyes C. Electro-remediación de Pb en suelos contaminados de Zimapán Hidalgo, usando agua y ácido acético como electrolitos. Universidad de Zimapán. 2010:1158-67. 21. Acar Y, Alshawabkeh A. Electrokinetic remediation 1. Pilot scale test with Pb-spiked kaolinite. J. . Geotech Eng 1996(122):173-85.

22. Reed B, Berg M, Thompson J, Hatfield J. Chemical conditioning of electrode reservoirs during electrokinetic soil flushing of Pb-contaminated silt. Environ Eng. 1995;1(121, 805-815.).

23. Hicks R, Tondorf S. Electrorestoration of metal contaminated soils. Environ Sci Technol. 1994(28):2203-10.

24. Suer P, Gitye K, Allard B. Speciation and transport of heavy metals and macroelements during electroremediation. . Environ Sci Technol. 2003;37:177-81.

25. Gristo P, Salvarrey A. Guía para la Identificación y Evaluación Preliminar de Sitios Potencialmente Contaminados Plan Nacional de Implementación del Convenio de Estocolmo. 2006;2(2).

26. García, Laura, Ramírez M, Reyes V. Electrorremediación de suelos arenosos contaminados por $\mathrm{Pb}, \mathrm{Cd}$ y As provenientes de residuos mineros, utilizando agua y acido acético como electrolitos. Sociedad Mexicana de Ciencias y Tecnología de Superficies y Materiales. 2011;24(1):24-9.

27. Salamanca AS, Sadeghian La densidad aparente y su relación con otras propiedades en suelos de la zona cafetera Colombiana. . Cenicafé 2015;56(4):381-97.

28. Ortiz Bea. Técnicas de recuperación de suelos contaminados Fundación para el conocimiento Madrid círculo, Huelva, España: . 2007;1(2):5-12.

29. Risco Cea. Electrokinetic Remediation of Soils Polluted with Pesticides. Flushing and Fence Technologies American Society of Civil Engineers, Reston, VA, España. 2016:20.

30. EPA. Soil Screening Guidance User 's Guide, Washington, DC USA 2016:1-39.

31. López A. Manual de Edafología Info agro Constanza. 2006.

32. Alcaino Concha, Guillermo Ignacio. . Análisis y comparación de tecnologías de remediación para suelos contaminados con metales Tesis pregrado Universidad de Chile. 2014.

33. Graciela S, Carmen P. Guía sobre suelos contaminados Conecta PYME Zaragoza, España. 2004. 34. De la Rosa Pérez D, ; Teurli, León; Ramírez, Marta, . Electrorremediación de suelos contaminados, una revisión técnica para su aplicación en campo Revista internacional de Contaminación Ambiental, Puebla, México. 2007;23:129-38.

35. Aragón C, De la Torre E, Guevara A. Diseño a escalas laboratorio y piloto de un sistema de remediación electrocinética de suelos contaminados con metales pesados Proyecto (tesis)(Pregrado) Escuela Politécnica Nacional, Quito, Ecuador. 2013:250.

36. Volke TV, Juan; De la Rosa, David. Suelos contaminados por metales y metaloides: muestreo y alternativas para su remediación Instituto Nacional de Ecología (INE-Semarnat). 2005.

37. Sanmiguel JX, Guerrero SR. Validación del método para determinar $\mathrm{Pb}, \mathrm{Cd}$, Ni por espectrometría de absorción atómica de llama en agua y suelo. . Escuela Superior Politécnica de Chimborazo, Orellana, Ecuador 2017. 2017:22-124.

38. AL-HAMDAN, Zahir A, Reddy; Krishna R. Transient behavior of heavy metals in soils during electrokinetic remediation. Chemosphere. 2008; United States of America vol. 71, no. 5, pp. 860871.

39. Gutiérrez PS, Roman de la Vara Análisis y diseño de experimentos. McGrawHill. 2008;2(3).

40. Reglamento Ambiental de Actividades Hidrocarburíferas. Decreto Ejecutivo 1215 (2010).

41. Herrera A. Efecto del silicio en la fertilidad del suelo, en la incidencia de enfermedades y el rendimiento del cultivo de arroz (Oryza sativa) var CR 4477. . Tecnológico de Costa Rica sede regional San Carlos, S1 2011. 2011;1:108.

42. Granda JF. Evaluación de la técnica de electrorremediación de suelos contaminados por metales pesados. Loja: Universidad Técnica Particular de Loja; 2017.

43. Pereira Cea. Edafología 1. Edafología Caldas. 2011. 